${ }^{2}$ Centro Hospitalar Entre Douro e Vouga, Rheumatology, Santa Maria da Feira, Portugal; ${ }^{3}$ Centro Hospitalar Universitário São João, Gastroenterology, Porto, Portugal

Background: Musculoskeletal symptoms represent the most common extraintestinal manifestations of inflammatory bowel disease (IBD) and a major cause of impaired quality of life in these patients. Spondyloarthritis $(\mathrm{SpA})$ is classically associated with IBD, but other rheumatic manifestations may occur.

Objectives: To characterize musculoskeletal symptoms and rheumatic diseases in an IBD cohort.

Methods: Retrospective monocentric descriptive study including all the patients with IBD consecutively reffered from Gastroenterology to the Rheumatology Department between January of 2013 and December 2020 in a tertiary university hospital. Demographic and clinical data and musculoskeletal symptoms were collected at the time of the first visit in the Rheumatology outpatient center and the rheumatic diseases diagnosed during the entire follow-up were registered. Results: A total of 234 patients were included, $136(58.1 \%)$ females, $20(8.5 \%)$ smokers. At the first Rheumatology consultation the mean age was $43.6( \pm 13.7)$ years and the mean IBD duration was $11.7( \pm 9.7)$ years. Concerning IBD: 172 (73.5\%) had Crohn's disease and $62(26.5 \%)$ had ulcerative colitis; azathioprine $(39.7 \%)$, infliximab $(28.2 \%)$ and mesalazine $(26.5 \%)$ were the most frequently used drugs; eleven patients (4.7\%) were taking glucocorticoids and $106(45.3 \%)$ had already been treated with glucocorticoids.

Regarding musculoskeletal symptoms: $76(32.5 \%)$ patients had peripheral symptoms and $98(41.9 \%)$ had axial symptoms (Table 1$)$

Table 1. Characterization of peripheral and axial musculoskeletal symptoms in patients with Inflammatory Bowel Disease.

\begin{tabular}{lcc}
\hline & & N (\%) \\
\hline Peripheral symptoms & No & $158(67.5 \%)$ \\
& Arthritis / "inflammatory" joint pain & $24(10.3 \%)$ \\
& "Mixed" rhythm joint pain & $15(6.4 \%)$ \\
& "Mechanical" joint pain & $29(12.4 \%)$ \\
Axial symptoms & Enthesopathy & $8(3.4 \%)$ \\
& No & $136(58.1 \%)$ \\
Total & "Inflammatory" back pain & $46(19.7 \%)$ \\
& "Mixed" rhythm back pain & $35(14.9 \%)$ \\
& "Mechanical" back pain & $17(7.3 \%)$ \\
& & $234(100 \%)$
\end{tabular}

Twenty-six (11.1\%) patients had radiographic sacroilitis, $14(6.0 \%)$ had sacroiliitis in computed tomography and $9(3.8 \%)$ in magnetic resonance. Forty-four (18.8\%) patients fulfilled Assessment of SpondyloArthritis international Society (ASAS) criteria for axial SpA and $5(2.1 \%)$ for peripheral SpA. Also of note, 16 (6.8\%) patients had a previous diagnosis of psoriasis and $5(2.1 \%)$ had uveitis in the past.

Concerning other rheumatic diagnosis, we observed: osteoarthritis in $64(27.3 \%)$ osteoporosis in $16(6.9 \%)$, diffuse idiopathic skeletal hyperostosis in $6(2.6 \%)$, systemic lupus erythematosus in $4(1.7 \%)$, rotator cuff tendinopathy in $2(0.9 \%)$, rheumatoid arthritis, gout, calcium pyrophosphate deposition disease, fibromyalgia, drug-induced lupus, osteitis condensans ilii, Dupuytren's contracture and avascular necrosis of the femoral head in $1(0.4 \%)$, each.

Conclusion: Our results demonstrate a high prevalence of musculoskeletal symptoms and rheumatic diseases in patients with IBD. These diagnoses are not limited to the group of SpA and osteoporosis, emphasizing the importance of rheumatologists being alert to other rheumatic diagnoses in patients with IBD. Disclosure of Interests: None declared

DOI: 10.1136/annrheumdis-2021-eular.490

\section{POS1271 PREVALENCE OF JOINT EFFUSION, HYPERPERFUSION AND ENTHESITIS IN YOUNG HEALTHY INDIVIDUALS}

J. K. Schreiner ${ }^{1}$, D. Scheicht ${ }^{2}$, P. Karakostas ${ }^{1}$, C. Behning ${ }^{3}$, P. Preuss ${ }^{4}$, P. Brossart ${ }^{1}$, V. Schäfer ${ }^{1} .{ }^{1}$ University Hospital Bonn, Clinic of Internal Medicine III, Oncology, Hematology, Rheumatology and Clinical Immunology, Bonn, Germany; ${ }^{2}$ Porz am Rhein Hospital, Department for Rheumatology, Cologne, Germany; ${ }^{3}$ University Hospital Bonn, Department of Medical Biometry, Informatics and Epidemiology, Bonn, Germany; ${ }^{4}$ University of Bonn, University Sport, Bonn, Germany

Background: The European League Against Rheumatism (EULAR) recommends using ultrasound (US) in patients with rheumatoid arthritis and psoriatic arthritis in diagnosis (1). In addition, US examination is crucial for detecting enthesitis in the above mentioned rheumatic diseases. However, abnormal findings on US may also occur in healthy individuals as indicated by recent studies focusing on small joints like metacarpophalangeal joint, metatarsophalangeal joint or the elbow. $(2,3)$
Ultrasound assessment of large joints in healthy individuals has not been extensively studied so far, causing a lack of information concerning normal values in musculoskeletal US examination.

Objectives: The purpose of this study was to determine the prevalence of joint effusion, hyperperfusion of the synovia and enthesitis in large and medium-sized joints of healthy individuals.

Methods: Ultrasound assessment of shoulder, elbow, wrist, hip, knee and ankle joints as well as corresponding entheseal sites including the Achilles tendon and the plantar aponeurosis was performed in healthy individuals below the age of 30 years. Additionally, participants filled out a survey on their physical activity level, underwent a bioelectrical impedance analysis (BIA) and conducted a supervised weight training to determine their training volume. Prevalence of US findings was calculated, and a binary logistic regression model was applied to determine the association between the present findings and sex, age, handedness, hours of sports activity per week, BMI, amount of skeletal muscle mass (SMM) or training volume of strength training.

Results: We included 51 healthy individuals with a mean age of 23.7 years, $52.9 \%$ were female with a mean BMI of $22.5 \mathrm{~kg} / \mathrm{m}^{2}$. Ultrasound examination detected joint effusion in one joint in $25.5 \%(n=13), 29.4 \%(n=15)$ showed effusion in two of twelve examined joints. Joint effusion in three, four and five joints was detected in $9.8 \%(n=5), 3.9 \%(n=2)$ and $3.9 \%(n=2)$ of the cases, respectively. In $27.5 \%(n=14)$ of the individuals no joint effusion was observed. The most frequently affected joint was the elbow joint. Synovial hyperperfusion was not detected in any participant. Enthesitis was observed in one, two and three examined entheseal sites in $19.5 \%(n=10), 5.9 \%(n=3)$ and $2 \%(n=1)$, respectively. In $72 \%(n=37)$ of the examined individuals no pathology of the entheseal sites was found. Hyperperfusion presented to be the most frequent pathology observed in enthesitis $(23 \%)$, followed by calcification $(6 \%)$.

Binary logistic regression model demonstrated a significant association between reported hours of sports activity per week and the prevalence of effusion in the knee $(p=0.017)$. The odds of joint effusion in the knee increased with the hours of sports activity by $34.1 \%(\operatorname{Exp}(B)=1.341,95 \%-\mathrm{Cl}(1.054,1.705))$. Additionally, the odds of enthesitis in any entheseal site increased with $B M I(p=0.015$, $\operatorname{Exp}(B)$ $=1.407,95 \%-\mathrm{Cl}(1.068,1.852)$.

Binary logistic regression model did not show any significant association between sex, age, handedness, amount of SMM or training volume of strength training and the prevalence of joint effusion or enthesis pathology in the examined participants.

Conclusion: Joint effusion in large and mid-sized joints as well as enthesitis are not only detected in patients with rheumatic diseases but also in healthy individuals. Hours of sports activity and BMI have a significant association with the findings and should be considered during ultrasound examination.

\section{REFERENCES:}

[1] Colebatch AN, Edwards CJ, Østergaard M, van der Heijde D, Balint PV D'Agostino M-A, u. a. EULAR recommendations for the use of imaging of the joints in the clinical management of rheumatoid arthritis. Ann Rheum Dis. Juni 2013;72(6):804-14.

[2] Padovano I, Costantino F, Breban M, D'Agostino MA. Prevalence of ultrasound synovial inflammatory findings in healthy subjects. Ann Rheum Dis. Oktober 2016;75(10):1819-23.

[3] Schäfer VS, Recker F, Vossen D, Ge I, Matuschek E, Hartung W. Prevalence of Elbow Joint Arthritis and Enthesitis in Rheumatoid Arthritis. 2020;11.

Disclosure of Interests: Julia Konstanze Schreiner: None declared, Dennis Scheicht: None declared, Pantelis Karakostas: None declared, Charlotte Behning: None declared, Peter Preuss: None declared, Peter Brossart: None declared, Valentin Schäfer Speakers bureau: AbbVie, Novartis, BMS, Chugai, Celgene, Medac, Sanofi, Lilly, Hexal, Pfizer, Janssen, Roche, Schire, Onkowissen, Royal College London, Consultant of: Novartis, Chugai, AbbVie, Celgene, Sanofi, Lilly, Hexal, Pfizer, Amgen, BMS, Roche, Gilead, Medac, Grant/research support from: Novartis, Hexal, Lilly, Roche, Celgene, Universität Bonn DOI: 10.1136/annrheumdis-2021-eular.1010

\section{POS1272 DIFFERENCES IN RANGE OF MOTION AND MECHANICAL MUSCLE CHARACTERISTICS AMONG INFLAMMATORY AND MECHANICAL SPINAL PAIN: A CASE-CONTROL STUDY}

S. Alcaraz-Clariana ${ }^{1}$, L. García-Luque ${ }^{1}$, I. C. Aranda-Valera ${ }^{2}$, M. L. Ladehesa Pineda $^{2}$, C. López-Medina ${ }^{2}$, C. Gonzalez-Navas ${ }^{3}$, F. Alburquerque Sendin ${ }^{1}$, D. P. Rodrigues-de-Sousa ${ }^{1}$, C. Garrido-González ${ }^{1}$, J. L. Garrido-Castro ${ }^{3}$, E. Collantes Estevez ${ }^{2}$ on behalf of CASTRO. ${ }^{1}$ University of Cordoba, Medicine, Nursing and Physiotherapy Faculty, Cordoba, Spain; ${ }^{2}$ Hospital Universitario Reina Sofia, Rheumatology, Córdoba, Spain; ${ }^{3}$ Maimónides Biomedical Research Institute of Córdoba, Motion Analysis Lab, Cordoba, Spain

Background: The spinal range of motion (ROM) and the mechanical muscle properties (MMPs) at rest are defined as relevant clinical factors in inflammatory (i.e. axial Spondyloarthritis -axSpA) and mechanical spinal pain (i.e. Mechanical 